\title{
A Comparison of Track-to-Track Fusion Algorithms for Automotive Sensor Fusion
}

\author{
Stephan Matzka and Richard Altendorfer
}

\begin{abstract}
In exteroceptive automotive sensor fusion, sensor data are usually only available as processed, tracked object data and not as raw sensor data. Applying a Kalman filter to such data leads to additional delays and generally underestimates the fused objects' covariance due to temporal correlations of individual sensor data as well as inter-sensor correlations. We compare the performance of a standard asynchronous Kalman filter applied to tracked sensor data to several algorithms for the track-to-track fusion of sensor objects of unknown correlation, namely covariance union, covariance intersection, and use of cross-covariance. For the simulation setup used in this paper, covariance intersection and use of cross-covariance turn out to yield significantly lower errors than a Kalman filter at a comparable computational load.
\end{abstract}

\section{INTRODUCTION}

Driver assistance systems (DAS) such as adaptive cruise control (ACC) or lane departure warning (LDW) are being offered by many car manufacturers and are getting more and more popular. While most current systems rely on only one exteroceptive sensor (e. g. radar or laser for ACC and camera for $\mathrm{LDW}$ ) in addition to proprioceptive sensors such as wheel speed and yaw rate sensors, enhanced versions of the above DAS such as ACC operating at the entire speed range or new systems such as autonomous braking for collision avoidance will require a more sophisticated exteroception that is based upon multiple sensors. Hence exteroceptive sensor fusion will play a crucial role for the performance of future DAS.

Automotive sensors such as radar are produced by automotive suppliers and usually output processed, tracked object lists. While a raw data interface might be provided by suppliers, hardware-specific, proprietary sensor knowledge is necessary to gain full advantage of raw sensor data. For DAS developed at car manufacturers, fusion of already tracked sensor objects is therefore the more likely type of data fusion.

In general sensor data from different sensor are output at different cycle times and are not synchronized to a common clock. The probably simplest level 1 fusion [9] of such sensor data would be achieved by an asynchronous Kalman filter for "sensor-to-track" fusion, where the fused object list of the Kalman filter is updated asynchronously every time a new sensor object list arrives. However, the application of Kalman filtering to such data is in principle incorrect since sensor objects of each sensor are temporally correlated due

S. Matzka is with Heriot-Watt University, Edinburgh, United Kingdom and with Ingolstadt University of Applied Sciences, Institute for Applied Research, 85049 Ingolstadt, Germany. (e-mail: sm217@hw.ac.uk)

R. Altendorfer is with TRW Automotive, 56070 Koblenz, Germany. (e-mail: richard.altendorfer@trw.com). to previous filtering and since tracked objects from different sensors are in general also correlated because of e. g. common modeling assumptions [3]. In addition, applying a Kalman filter to already (Kalman-)filtered data will result in additional phase delays due to the Kalman filter's low-pass characteristics.

In order to overcome the above difficulties several track-totrack fusion algorithms have been proposed. In this paper we focus on three well-established methods, namely use of crosscovariance [1], covariance intersection [6], and covariance union [11]. The goal of this paper is to assess the improvement in tracking accuracy by those algorithms with respect to an asynchronous Kalman filter that is often used despite its known shortcomings, see e. g. [12]. By comparing the root mean squared errors (RMSE) and correlation coefficients for simulated trajectories of the four aforementioned fusion strategies, we want to quantify in detail an assessment of trackto-track fusion algorithms briefly mentioned in [4].

The paper is organized as follows: in section II we present an overview of our simulation setup. In section III we briefly review the track-to-track fusion algorithms to be used. Section IV contains a detailed discussion of the simulation results. Details of the simulation setup such as the vehicle dynamics or the sensor model are relegated to the appendix.

\section{Simulation SETUP}

In order to assess different fusion strategies by numerical simulation, the following subsystems are needed: the generation of a reference trajectory for the target vehicle to be observed by sensors, the simulation of the sensor measurements and the sensor processing (Kalman filter), implementations of the abovementioned "sensor"-to-track fusion and track-totrack fusion algorithms and an error computation module that compares the fusion results to ground truth - the reference trajectory. In this setup we restrict ourselves to a single target vehicle in order to cleanly assess the performance of the fusion algorithms without additional errors due to false associations. Furthermore, the simulated scenario is static in the sense that the ego vehicle on which the sensors are mounted does not move; hence no "ego-compensation" is necessary and the relative measurements that the sensors provide are also measurements in an inertial system whose origin is taken to be at the ego vehicle. A block diagram of the simulation setup is shown in Fig. 1. 


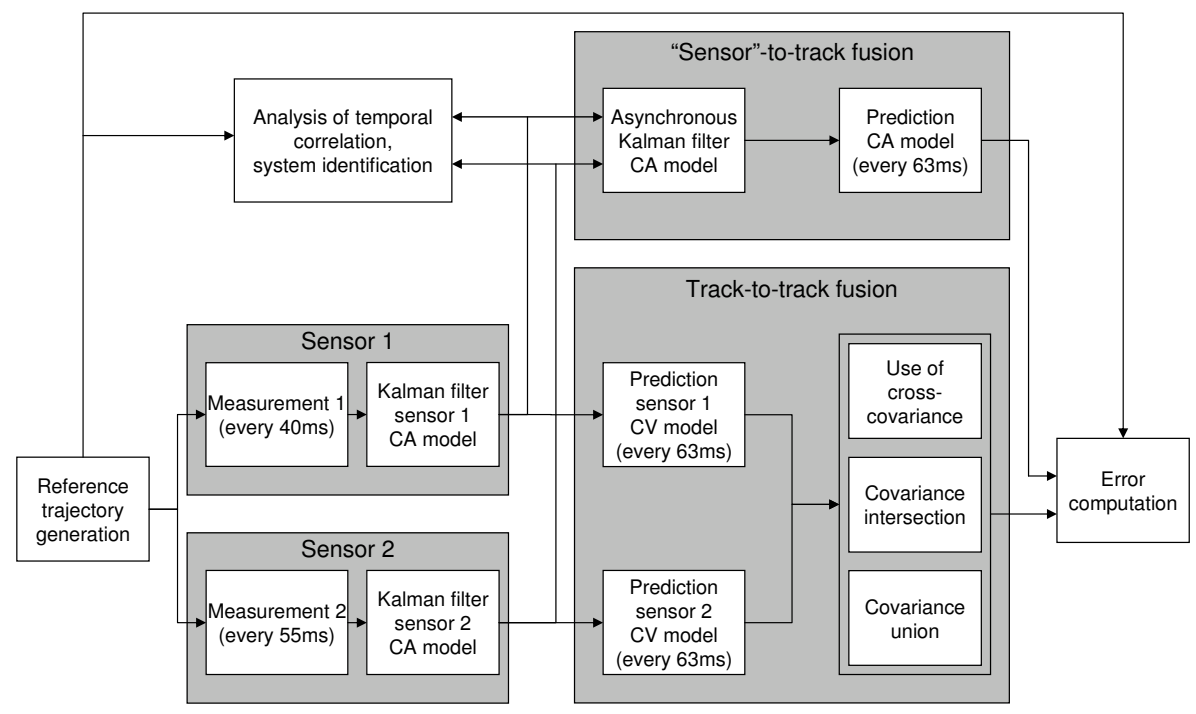

Fig. 1. Block diagram of the simulation setup. CA (constant acceleration) model refers to a white noise jerk model and CV (constant velocity) model refers to a white noise acceleration model both detailed in the appendix.

\section{A. Reference trajectory generation}

The trajectory of the target vehicle is generated with a $2 \mathrm{D}$ white noise jerk model, for details see app. A. It is run at a cycle time of $1 \mathrm{~ms}$. With process noise covariance values of $10\left(\frac{m}{s^{3}}\right)^{2}$, trajectories are the result of random walk dynamics and tend to proceed in a relatively straight line. If the target leaves a rectangular region of $\pm 200 \mathrm{~m}$ in $\mathrm{x}$-direction and \pm $150 \mathrm{~m}$ in $\mathrm{y}$-direction around the centre, a constant bias of

$$
\begin{aligned}
& \dddot{x}=-\operatorname{sgn}(x) \cdot 10 \frac{m}{s^{3}}, \text { if }|\mathrm{x}|>200 \mathrm{~m} \\
& \dddot{y}=-\operatorname{sgn}(y) \cdot 10 \frac{m}{s^{3}}, \text { if }|\mathrm{y}|>150 \mathrm{~m}
\end{aligned}
$$

is superimposed on the white noise jerk in order to force the object back into the rectangular region. The repulsive force is applied as long as the trajectory proceeds along its path away from the centre and is outside the defined rectangular region.

Parts of the vehicle trajectory where this non-random jerk component is applied are crucial in assessing the different fusion algorithms' performances since the underlying dynamical models of the various Kalman filters in this simulation setup will not be appropriate in these cases.

\section{B. Sensor simulation}

Measurements of the two sensors are assumed to be the relative position of the target vehicle with respect to the ego vehicle as would be appropriate for a laser scanner, for example. The measurements are corrupted by Gaussian noise and are then fed into a Kalman filter with a white noise jerk model, for details see app. B. For a realistic sensor scenario, the two sensors output data at different cycle times of $40 \mathrm{~ms}$ and $55 \mathrm{~ms}$.

\section{C. "Sensor"-to-track fusion}

The "sensor"-to-track fusion of the two sensors is achieved by an asynchronous Kalman filter, i. e. a Kalman filter that updates its state every time a new measurement arrives. This Kalman filter - like the sensor Kalman filters - also uses a white noise jerk model (see app. A). In our setup we assume that automotive applications that use sensor fusion data require fused objects at a constant cycle time of $63 \mathrm{~ms}$. Hence a prediction module using the same dynamical model as the Kalman filter is activated every time an output is required by the application. Kalman filter and prediction module together constitute the "sensor"-to-track fusion module.

\section{Track-to-track fusion}

In track-to-track fusion, the states to be fused must be synchronized, i. e. must have the same time stamp. In general, however, sensors output data at different cycle times with local, non-synchronized clocks. While synchronization methods of various degrees of sophistication have been proposed in literature [2], we use the simplest one, namely prediction of all sensor data to the time required by the application. Since the details of sensor preprocessing and tracking are usually kept confidential by automotive suppliers, we make the conservative choice of a white noise acceleration model for the prediction inside the track-to-track fusion module. The synchronized sensor data are then fed into one of the trackto-track fusion algorithms under consideration, namely use of cross-covariance, covariance intersection, and covariance union. A brief review of those three algorithms is provided in section III.

\section{E. Error computation}

Every $63 \mathrm{~ms}$ the asynchronous Kalman filter and the three track-to-track fusion algorithms output fused target state vec- 
tors and their covariances. Using the target vehicle reference trajectory the RMSE can be computed at every time step. We then average the RMSE of the position coordinates over entire simulation runs of $40 \mathrm{~s}$. This allows us to evaluate the benefits of track-to-track fusion versus Kalman filtering when the dynamical model of the Kalman filter is valid and when it is not.

\section{TRACK TO TRACK FUSION}

\section{A. Use of Cross-Covariance}

The use of cross-covariance method was first described in [1]. Given two state estimates $\widehat{\xi_{a}}$ and $\widehat{\xi_{b}}$ and their covariance matrices $P_{a}$ and $P_{b}$, the fused estimate using cross-covariance reads

$$
\widehat{\xi_{c}}=\widehat{\xi_{a}}+\chi\left[\widehat{\xi_{b}}-\widehat{\xi_{a}}\right]
$$

where

$$
\begin{gathered}
\chi=\left[P_{a}-P_{a b}\right] U_{a b}^{-1} \\
U_{a b}=P_{a}+P_{b}-P_{a b}-P_{a b}^{T}
\end{gathered}
$$

The covariance matrix for the estimation pair is calculated using

$$
P_{c}=P_{a}-\left[P_{a}-P_{a b}\right] U_{a b}^{-1}\left[P_{a}-P_{a b}\right]^{T}
$$

The cross-covariance matrix $P_{a b}$ is initially set to $P_{a b}(0 \mid 0)$ and subsequentially updates using a recursive relationship

$$
P_{a b}(k \mid k)=\alpha_{a}(k) \beta(k-1) \alpha_{b}^{T}(k)
$$

where

$$
\begin{gathered}
\alpha_{a}(k)=I-K_{a}(k) H_{a}(k), \alpha_{b}(k)=I-K_{b}(k) H_{b}(k) \\
\beta(k-1)=F_{a} P_{a b}(k-1 \mid k-1) F_{b}^{T}+Q(k-1)
\end{gathered}
$$

However, for $\alpha_{a}(k)$ and $\alpha_{b}(k)$ in (5) the details of both sensors' Kalman filters such as $H_{i}, K_{i}$, and $F_{i}$ as well as $Q$ have to be known. This is not always the case, especially for industrial sensors with integrated tracking. For this case, an alternative computation of $P_{a b}$ is proposed in [2] where the cross-covariance matrix is approximated by the Hadamard (or entrywise) product of both input covariance matrices.

$$
P_{a b}=\rho \sqrt{P_{a} \bullet P_{b}}
$$

where $\rho$ represents an effective correlation coefficient. It can for example be determined numerically by Monte-Carlo simulation for specific setups; in [2] the value $\rho \approx 0.4$ was found to be a good approximation for typical tracking situations.

\section{B. Covariance Intersection}

In [6], [10] the use of a fusion rule named Covariance Intersection (CI) for combining tracks of unknown correlation is proposed.

Based upon two estimates $A$ and $B$ that can originate both from a sensor or a model of the observed process it is possible to determine a fused estimate $C$. For consistent estimates $A, B$, it is possible to represent these as a pairs of estimated state and covariance $A=\left\{\widehat{\xi_{a}}, P_{a}\right\}$ and $B=\left\{\widehat{\xi_{b}}, P_{b}\right\}$. Note that these pairs do not contain any information about the cross correlation between $A$ and $B$.

The fused estimate $C$ can then be determined using

$$
\begin{gathered}
P_{c}^{-1}=\omega P_{a}^{-1}+(1-\omega) P_{b}^{-1} \\
\widehat{\xi_{c}}=P_{c}\left(\omega P_{a}^{-1} \widehat{\xi_{a}}+(1-\omega) P_{b}^{-1} \widehat{\xi_{b}}\right)
\end{gathered}
$$

where

$$
\omega=\arg \min \left(\operatorname{det}\left(P_{c}\right)\right)
$$

The computation of $\omega$ in (9) is determined through an optimisation process to minimise $\operatorname{det}\left(P_{c}\right)$, which is proposed in [7]. Yet is is also possible to minimise $\operatorname{tr}\left(P_{c}\right)$ or other criteria of uncertainty. For all $\omega \in[0,1]$ the consistency of the estimate is guaranteed. Moreover, in [10] this estimate is shown to be optimal if the cross-covariance is unknown.

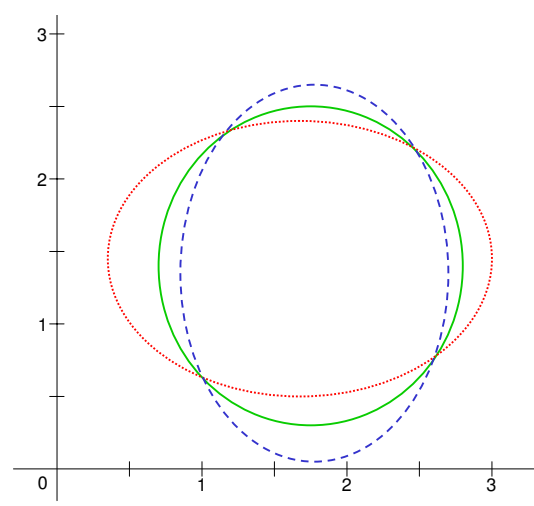

Fig. 2. Covariance ellipses (locus of points $x^{T} P^{-1} x=1$ ) of $A$ (dotted red ellipse) and $B$ (dashed blue ellipse) and their covariance intersection fusion result $C$ (solid green ellipse) for $\omega=0.5$.

\section{Covariance Union}

A common problem in track-to-track data fusion is the resolution of statistically inconsistent states - known as database deconfliction. The covariance union method proposed in [11] allows to unify two tracks, even if the difference of the state estimates exceeds the covariance indicated by at least one track. To obtain a unified estimate, a new state vector estimate $\widehat{\xi}_{c}$ is determined. The unified covariance matrix $P_{c}$ is a covariance exceeding both $P_{a}$ and $P_{b}$.

The unified estimate $C=\left\{\widehat{\xi}_{c}, P_{c}\right\}$ can be determined by 


$$
\begin{gathered}
U_{a}=P_{a}+\left(\widehat{\xi_{c}}-\widehat{\xi_{a}}\right) \cdot\left(\widehat{\xi}_{c}-\widehat{\xi_{a}}\right)^{T} \\
U_{b}=P_{b}+\left(\widehat{\xi_{c}}-\widehat{\xi_{b}}\right) \cdot\left(\widehat{\xi_{c}}-\widehat{\xi_{c}}\right)^{T} \\
P_{a b}=\max \left(U_{a}, U_{b}\right)
\end{gathered}
$$$$
\widehat{\xi}_{c}=\arg \min \left(\operatorname{det}\left(P_{a b}\right)\right)
$$

As for the covariance intersection method an optimisation process has to be performed to minimise a criterion of uncertainty such as determinant, trace, etc. For the covariance union method, $\widehat{\xi}_{c}$ has to be determined, which represents a multidimensional optimisation problem. In our simulation setup, we optimise $\widehat{\xi}_{c}=(x y)^{T}$.

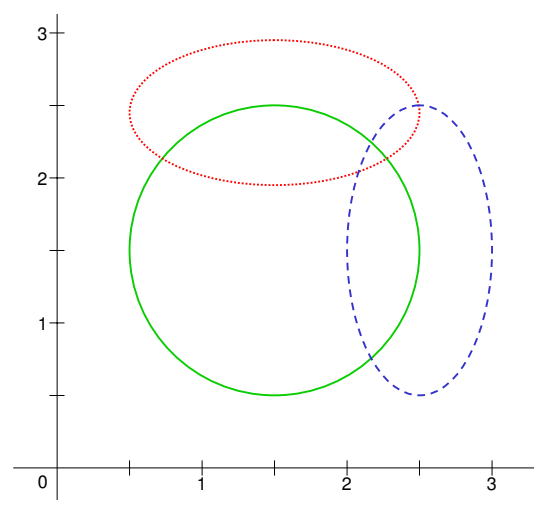

Fig. 3. Example of a covariance union for $P_{a}$ (dotted red ellipse) and $P_{b}$ (dashed blue ellipse). For $\widehat{\xi_{u}}=\left(\begin{array}{ll}1 & 1\end{array}\right)^{T} \operatorname{det}\left(P_{c}\right)$ is minimal (solid green ellipse).

\section{Computational costs of track-to-track fusion}

Computational costs for the asynchronous Kalman filter and the track-to-track fusion algorithms are measured in our simulation setup running on a $2 \mathrm{GHz}$ Pentium IV processor in eight test runs of 10,000 updates and predictions for each method. The computation times are given in Table I as well as the relative time per update and prediction as compared to the asynchronous Kalman filter.

\begin{tabular}{|l||c|c|}
\hline Method & absolute [ns] & relative \\
\hline \hline Async. Kalman & 151.75 & 1.00 \\
Use of cross cov. & 103.95 & 0.69 \\
Cov. intersection & 132.30 & 0.87 \\
Cov. union & 397.50 & 2.62 \\
\hline
\end{tabular}

TABLE I

MEAN ABSOLUTE AND RELATIVE COMPUTATION TIMES FOR THE ASYNCHRONOUS KALMAN FILTER AND THE TRACK-TO-TRACK FUSION ALGORITHMS.

The measured computational costs in Table I show that the use of cross covariance approach performs at the smallest computational cost, followed by covariance intersection. The asynchronous Kalman filter requires slightly more computational resources, but far less than the covariance union method. Note that the multi-dimensional optimisation for the covariance union has been implemented in a preliminary way using simple gradient descent. It can clearly be sped up using more sophisticated multi-dimensional minimization techniques such as Nelder-Mead, etc.

\section{Evaluation}

Evaluation of the track-to-track fusion algorithms discussed in section III is performed using our simulation setup. In the following two effects are discussed: the effect of sensor crosscorrelation and the sensors' auto-correlation.

\section{A. Cross Correlation}

The adverse effect of correlated input sources is a major problem in track-to-track fusion. We use our simulation setup to evaluate the performance of three different track-totrack fusion algorithms and compare their performances to that of a standard asynchronous Kalman filter. Towards this end, we determine the RMSE at the outputs of both sensor Kalman filters. The inter-sensor (track-to-track) correlation of the errors is computed numerically over entire simulation runs by comparing the sensor outputs to the reference trajectories. Track-to-track fusion results are evaluated in the error computation module by comparing the reference trajectory to the fusion results. The resulting overall RMSE values for an individual test run are plotted against the measured track-totrack correlation in Fig. 4

It can be seen from Fig. 4 that the asynchronous Kalman filter performs well, that is with a lower RMSE value than both input tracks, for most test runs with a cross-correlation $\rho<0.15$. However, for input tracks with a correlation $\rho>0.15$, the fusion errors become higher than the RMSE of both input vectors. On the other hand the errors of the trackto-track fusion methods are smaller than those of the Kalman filter with the exception of covariance intersection at small correlations; their range is also smaller.

\begin{tabular}{|l||c|c|c|}
\hline Method & Overall & $-0.2<\rho<0.2$ & $0.2<\rho<0.6$ \\
\hline \hline Async. Kalman & $0.283(0.317)$ & $0.181(0.138)$ & $0.369(0.358)$ \\
Use of cross cov. & $0.151(0.150)$ & $0.131(0.129)$ & $0.167(0.164)$ \\
Cov. intersection & $0.189(0.187)$ & $0.178(0.176)$ & $0.199(0.198)$ \\
Cov. union & $0.156(0.156)$ & $0.137(0.134)$ & $0.172(0.170)$ \\
\hline
\end{tabular}

TABLE II

MEAN (MEdian) Root MEAN SQUaRed ERRor of ALl TEST RUNS, TEST RUNS WITH LOW CROSS-CORRELATION AND HIGH-CROSS CORRELATION.

The mean and median RMSE values for 2000 test runs over 40s can be seen from Table II. Considering the mean RMSE values, the use of cross covariance algorithm yields the lowest RMSE, followed by covariance union, covariance intersection, and finally the asynchronous Kalman filter.

\section{B. Auto Correlation}

Apart from the cross-correlation between the two Kalman filter tracks, the auto-correlation of each Kalman track is determined numerically assuming ergodicity. The auto-correlation 

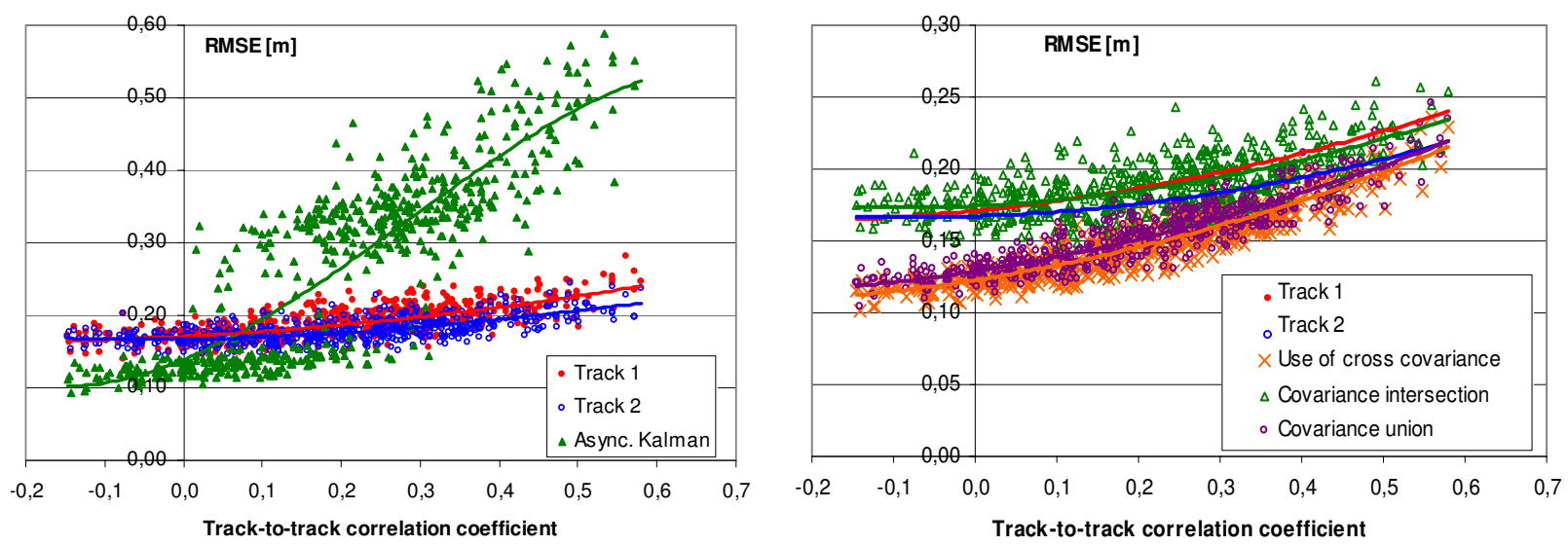

Fig. 4. Root mean squared errors (RMSE) of track fusion results and input tracks Track 1 and Track 2 depending on the cross correlation of the latter. Due to considerably differing RMSE values of the asynchronous Kalman filter and the other fusion algorithms two diagrams with differing RMSE ranges are used.

for the position and velocities of both Kalman sensor tracks is drawn against the time difference in Fig. 5.

The auto-correlation plot in Fig. 5 is indicative of a second order Gauss-Markov process, as is expected from a second order (white noise jerk model) Kalman filter. The degree of auto-correlation is significant since even after $0.25 \mathrm{~s}$ the autocorrelation coefficient exceeds 0.5 for both sensors.

Therefore the assumption of temporal independence does not hold for the Kalman filter tracks which are used in the the track-to-track fusion process. This property does not affect the discussed track-to-track fusion algorithms as these perform the fusion exclusively on the most actual track updates. However, the asynchronous Kalman filter assumes temporal independence which is clearly not the case for the given setup. These problems have been addressed in literature, i.e. in [5] the use of an augmented dynamical model is proposed to handle temporal correlation.

\section{Conclusions And Outlook}

We presented an assessment of a standard Kalman filter applied to tracked sensor data of unknown correlation versus several well-known algorithms for track-to-track fusion. Tracked sensor data were evaluated for inter-track correlation as well as for auto-correlation.

Among the three track-to-track algorithms, the use of crosscovariance returned the best quality of fusion results with respect to RMSE. Moreover, the run time of the use of cross-covariance was the lowest of the four fusion methods. Covariance intersection required the second fastest runtime with a RMSE that was lower than the Kalman filter but higher than both use of cross covariance and covariance union. The covariance union algorithm showed RMSE values comparable to the use of cross-correlation albeit at a considerably higher computational cost. The standard Kalman filter performs well for small inter-track cross-correlations however for large crosscorrelations the RMSEs are a factor of 2-3 larger than for the track-to-track correlation methods. Its computational cost is between those of use of cross-covariance and covariance intersection, and the cost for covariance union.

The poor performance of the Kalman filter at large correlations was expected. More interesting is the ranking of the track-to-track fusion methods with respect to RMSE: as was observed in [7], covariance intersection is a conservative fusion method that can be improved if partial knowledge of crosscovariance can be incorporated. Hence the better results for the use of cross-covariance. Perhaps more unexpectedly, covariance union yields results better than covariance intersection and comparable to those of use of cross-covariance. Due to the application of non-random jerk during the vehicle motion (cf. section II-A) statistically inconsistent sensor states ${ }^{1}$ might arise that are better taken care of by covariance union than by covariance intersection.

The auto-correlation of both sensor tracks have been evaluated to be significant, exceeding 0.9 for a single fusion cycle of $40 \mathrm{~ms}$ and $55 \mathrm{~ms}$, respectively. Auto-correlation does not affect the track-to-track fusion algorithms investigated in this paper, as they do not include past states and covariances. The detrimental effect of autocorrelated measurements on the Kalman filter is due to its assumption of the whiteness of the measurement noise. The Kalman filter can, however, be improved by using an augmented state that includes a model of the autocorrelation dynamics [5]. The exact system identification and its incorporation into the Kalman filter constitutes future work.

While the numerical study presented in this paper gives an indication of performance improvements using track-totrack fusion for automotive applications, many simplifying assumptions about vehicle dynamics, sensor characteristics, sensor filtering, etc have been made. The next step will be the implementation of the above track-to-track algorithms in a test vehicle and their performance assessment in realistic traffic scenarios using real sensor data.

\footnotetext{
${ }^{1}$ Statistical inconsistency of two states representing the same physical entity can for example be characterized by a threshold for the Mahalanobis distance (cf. [11]).
} 


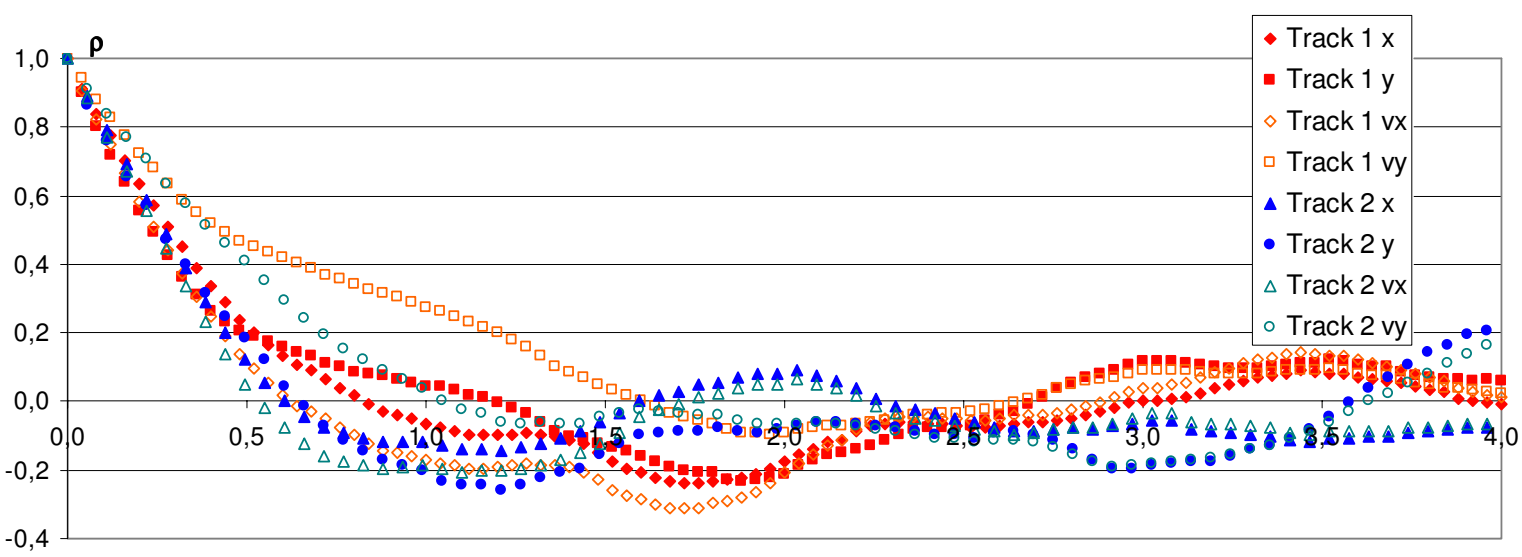

$\delta t[s]$

Fig. 5. Auto-correlation coefficient $\rho$ of Kalman filter tracks Track 1 and Track 2 respectively drawn against the time difference $\delta t$.

\section{APPENDIX}

\section{A. Vehicle dynamics}

The reference vehicle trajectory is characterized by a sixdimensional state vector

$$
\xi=\left(\begin{array}{llllll}
x & y & \dot{x} & \dot{y} & \ddot{x} & \ddot{y}
\end{array}\right)^{\top}
$$

The dynamical model is a discrete-time counterpart white noise jerk model [8]

$$
\xi(k+1)=F(k+1, k) \xi(k)+G(k+1, k) \nu(k)
$$

where

$$
\begin{aligned}
F(k+1, k)= & \left(\begin{array}{cccccc}
1 & 0 & \Delta t_{k} & 0 & \frac{\Delta t_{k}^{2}}{2} & 0 \\
0 & 1 & 0 & \Delta t_{k} & 0 & \frac{\Delta t_{k}^{2}}{2} \\
0 & 0 & 1 & 0 & \Delta t_{k} & 0 \\
0 & 0 & 0 & 1 & 0 & \Delta t_{k} \\
0 & 0 & 0 & 0 & 1 & 0 \\
0 & 0 & 0 & 0 & 0 & 1
\end{array}\right) \\
G(k+1, k)= & \left(\begin{array}{cc}
\frac{\Delta t_{k}^{3}}{6} & 0 \\
0 & \frac{\Delta t_{k}^{3}}{6} \\
\frac{\Delta t_{k}^{2}}{2} & 0 \\
0 & \frac{\Delta t_{k}^{2}}{2} \\
\Delta t_{k} & 0 \\
0 & \Delta t_{k}
\end{array}\right)
\end{aligned}
$$

with $\Delta t_{k}=t_{k+1}-t_{k}$. The two-dimensional stochastic process vector $\nu(k) \in \mathbb{R}^{2}$ models the process noise of the vehicle dynamics. It is a white, Gaussian process with $\operatorname{cov}(\nu(k))=$ $V=\operatorname{diag}(10,10)\left(\frac{m}{s^{3}}\right)^{2} \quad \forall k$. In our simulation the state $\xi$ is updated every $1 \mathrm{~ms}$ using (15).

This model is also referred to as CA model in Fig. 4. The $\mathrm{CV}$ model used in the track-to-track predictions is a truncated CA model.

\section{B. Sensor measurements}

Sensors 1 and 2 are assumed to measure position only, hence the output equation for the Kalman filter reads

$$
z_{i}(k)=H(k) \widehat{\xi}_{i}(k)+w_{i}(k) \quad i \in\{1,2\}
$$

where $z_{i}(k)$ are the measurements, $\widehat{\xi}_{i}(k)$ are the internal states of the sensor Kalman filters and $w_{i}(k)$ are two-dimensional stochastic processes simulating the sensor noise. The output function is given by

$$
H(k)=\left(\begin{array}{cccccc}
1 & 0 & 0 & 0 & 0 & 0 \\
0 & 1 & 0 & 0 & 0 & 0
\end{array}\right)
$$

The noise processes are characterized by

$$
\begin{aligned}
& \operatorname{cov}\left(w_{1}(k)\right)=W_{1}=\operatorname{diag}(0.25,0.25) m^{2} \quad \forall k \\
& \operatorname{cov}\left(w_{2}(k)\right)=W_{2}=\operatorname{diag}(0.16,0.09) m^{2} \quad \forall k
\end{aligned}
$$

\section{REFERENCES}

[1] Y. Bar Shalom, "On the track-to-track correlation problem," IEEE Transactions on Automatic Control, vol. AC-26, no. 2, pp. 571-572, 1981.

[2] Y. Bar-Shalom and X.-R. Li, Multitarget-multisensor tracking: Principles and techniques. YBS Publishing, 1995.

[3] S. Blackman and R. Popoli, Design and analysis of modern tracking systems, 2nd ed., ser. Artech House radar library. Artech House, 1999.

[4] N. Floudas, M. Tsogas, A. Amditis, and A. Polychronopoulos, "Track level fusion for object recognition in road environments," PReVENT Fusion Forum e-Journal, no. 2, pp. 16-23, January 2008.

[5] A. Gelb, Ed., Applied optimal estimation. MIT Press, 1974, ch. 4.5, pp. $133-136$.

[6] S. J. Julier and J. K. Uhlmann, Handbook of Data Fusion. CRC Press, 2001, ch. 12: General decentralized data fusion with covariance intersection (CI), pp. 1-25.

[7] - "Using covariance intersection for slam," Robotics and Autonomous Systems, vol. 55, no. 1, pp. 3-20, 2007.

[8] X. R. Li and V. P. Jilkov, "Survey of maneuvering target tracking-part I: dynamic models," IEEE Transactions on Aerospace and Electronic Systems, vol. 39, no. 4, pp. 1333-1364, 2003.

[9] A. N. Steinberg, C. L. Bowman, and F. E. White, "Revisions to the JDL data fusion model," in Proceedings of SPIE, Sensor Fusion: Architectures, Algorithms, and Applications III, vol. 3719, 1999, pp. 430-441.

[10] J. K. Uhlmann, "Dynamic map building and localization for autonomous vehicles," Ph.D. dissertation, University of Oxford, 1995.

[11] - "Covariance consistency methods for fault-tolerant distributed data fusion," Information Fusion, vol. 4, no. 3, pp. 201-215, 2003.

[12] K. Weiss, D. Stueker, and A. Kirchner, "Target modeling and dynamic classification for adaptive sensor data fusion," in Proceedings of the IEEE Intelligent Vehicles Symposium, 2003, pp. 132-137. 